\title{
Identifying association of a STIM1 genes related to prognosis of solid tumor: a bioinformatics analysis of gene expression data
}

\author{
Md Misbah ${ }^{1,2}$, Wei Chiao Chang ${ }^{1,2}$ \\ ${ }^{I}$ Clinical Pharmacy, Taipei Medical University, Taiwan, ${ }^{2}$ Master Program for Clinical Pharmacogenomics and \\ Pharmacoproteomics, Taipei Medical University, Taiwan
}

\section{Abstract}

Background: Store operating calcium channels (SOCCs) mediate calcium influx across the intracellular and Endoplasmic reticulum of the Cell. SOCCs is a very important for human physiological function that regulates events including cell survival, cell proliferation and gene transcription of tumour tissues. In the case of signalling events downstream of SOCCmediated calcium influx are not fully confirmed, but it is clear that variations in the expression of SOCCs promote tumour formation and obstruct response to treatment.

Methods: We evaluated the expression of SOCC genes (all two subtypes; STIM1 and ORAI1) and their prognostic value in four major solid tumours (i.e. gastric, lung, breast and ovarian cancers) via a publicly accessible database system.

Results: In gastric cancer, expression of all the SOCC genes was related with overall survival (OS) among stage I-IV patients $($ all $\mathrm{p}<0.05)$. By combining the two potential biomarkers, a SOCC signature was developed, which retained a significant association with OS in all stage patients. In lung and ovarian cancer, association with OS was also significant when all tumour stages were considered, but was partly lost or inconclusive after splitting cases into localized and metastatic subsets.

Conclusions: Alterations in STIM1 gene expression are linked to tumour prognosis. Gastric cancer represents the most promising setting for further evaluation. 